\title{
La atención primaria en la encrucijada: desplegar su potencial transformador o quedar instrumentados en una nueva ofensiva privatizadora
}

\author{
Primary care at the crossroads: deploy its transforming potential or \\ be instrumented in a new privatization offensive
}

Mario Rovere'

DOI: 10.1590/0103-11042018S121

RESUMEN Luego de cuarenta años de instalada en el firmamento sanitario, la Atención Primaria de la Salud (APS) se encuentra como nunca antes frente a la posibilidad de ser reescrita. No se trata de esos intentos de reafirmación o ampliación que se vivieron a los 25 (Organización Panamericana de la Salud - OPS) o a los 30 años (Organización Mundial de la Salud - OMS); se trata de un intento de reeditar los gestos y los símbolos de la histórica reunión que ocurriera en Alma Ata. El contexto internacional es diferente al de 1978, por lo que existen fundados temores que la nueva declaración constituya un retroceso respecto a la Declaración original. Al menos el primer borrador que la OMS puso a circular refuerza esa preocupación al subsumir la APS a la controversial iniciativa de la OMS y del Banco Mundial: cobertura universal en Salud. Como antes y como siempre el resultado final dependerá más bien de la forma como las comunidades y los trabajadores de salud reinterpreten la propuesta en cada lugar concreto.

PALABRAS-CLAVE Políticas públicas de salud. Defensa de la salud. Atención Primaria de Salud. Salud global.

ABSTRACT After 40 years of settlement in the sanitary firmament the Primary Health Care (PHC) faces, as never before, the possibility to be re written. It isn't the same context when PHC was reaffirmed as in the 25th anniversary (Pan American Health Organization - Paho) or widen as in its 30th anniversary (World Health Organization-WHO); instead of it, we see an intent to re-edit the gestures and the symbols of those historical meeting of Alma Ata in 1978, but this time, it will take place in the city of Astana. The international context couldn't be more different from those in 1978. That's why it exists funded fears that the new statement will become a backward from the strength of the original statement. Unless this is what we can presume after reading

1 Universidad Nacional de Rosario, Centro de Estudios Interdisciplinarios, Maestría en Salud Pública

- Rosario, Argentina. Orcid: https://orcid. org/0000-0002-64132120

roveremarior@gmail.com the first paper of WHO for this meeting where it tries to subsume PHC to Universal Health Coverage, a controversial initiative of WHO and the World Bank. As it was and it always be, the final outcome, in fact, will depend of the way the communities and the health workers translate the proposal to every concrete practice scenario.

KEYWORDS Public health policy. Health advocacy. Primary Health Care. Global health. 
Para quien vivencia la atención primaria y se impregna de ella, deja de ser la intelección de un concepto y pasa a ser un sentimiento.

(J. Monsalvo)

\section{Introducción}

Resulta bastante complejo asumir el desafío de escribir algo original o que pueda, en algún sentido, aportar a la enorme cantidad de publicaciones que el campo de la atención primaria ha generado en sus 40 años de existencia formal.

Al mismo tiempo resultaría literalmente imposible intentarlo y comenzar cualquier análisis crítico, que sea al mismo tiempo propositivo sobre la Atención Primaria de la Salud (APS), sin explicitar una fuerte valoración a los cientos de miles de trabajadores comunitarios, administrativos, auxiliares, técnicos y profesionales que en todo el mundo se han apropiado de este nombre, lo han resignificado, adecuándolo a cada realidad concreta, y que orienta su quehacer cotidiano en el sentido del derecho a la salud de las poblaciones más postergadas.

En contraste con esta evidencia se podrá igualmente argumentar que siempre esta tarea resulta y resultará insuficiente porque la máquina de producir inequidades en el mundo y en cada una de nuestras sociedades no deja ni dejará de concentrar la riqueza y con ello no (dejará) de generar su cuota creciente de exclusión y de violencia.

Quizás un punto intermedio de reflexión, alejado del idealismo pero también del puro determinismo, sea preguntarnos: ¿qué situación enfrentarían hoy los sectores populares en el mundo, sin esta mezcla de permanente construcción, de conquista con avances y retrocesos y de intentos periódicos de remodelación parcial de los sistemas de salud al que por 40 años hemos denominado APS?

A pesar de las críticas, de las insuficiencias, de los embates, de las simplificaciones, o de las reducciones, la APS se instaló en las prácticas, en los discursos y en los idearios del sector salud a nivel global, pero paradójicamente apelando casi como identidad y como motor a la fuerza de lo local.

Varias décadas después algunos operadores de la salud mundial buscan de algún modo, canibalizarla, domesticarla y refuncionalizarla de acuerdo a sus propios propósitos. De esta manera que lo que en su momento se pensó como el motor para la construcción de sistemas nacionales de salud equitativos y como un dispositivo diseñado y dirigido para el derecho a la salud, pase ahora a convertirse en la punta de lanza para la última travesura del complejo médico industrial y del neoliberalismo en salud: la engañosamente denominada Cobertura Universal en Salud o CUS.

No se trata de especulaciones: el borrador de la Organización Mundial de la Salud (OMS) que se busca consensuar en Astana, Kazajstan, en octubre del 2018, para actualizar la APS del Siglo XXI es taxativo al respecto:

[...] lanzamos un movimiento global en post de la cobertura universal de salud [...], asegurando que la Atención Primaria de Salud se encuentre en el centro de este movimiento ${ }^{1(1)}$.

\section{Contextualizando el surgimiento de la APS}

Las condiciones materiales, económicas, políticas y sociales resultan fundamentales a la hora de constituir expectativaticas. No se trata de una abstracción, el informe del World Inequality Lab² (WIL) es contundente mostrando hasta qué punto la declaración de Alma Ata se escribió en un momento único de la historia reciente: un momento de mejora relativa de los términos de intercambio entre los países impulsada por la 
experiencia del club de países exportadores de petróleo que abrigaba la esperanza de un Nuevo Orden Económico Internacional más justo, e incluso una mejora en la redistribución del ingreso al interior de los países más desarrollados que en un ciclo largo, de más de un siglo, muestra a la década del 19751985 como la más equitativa en distribución del ingreso (1913-2015).

Es que la bipolaridad no era solo un tema de política internacional, también al interior de los propios países alimentaba una razonable expectativa de sostener un equilibrio o hasta un pacto de clases, base de los sistemas de Estado de Bienestar en regímenes políticos que se balanceaban entre el capitalismo y el socialismo.

En el esquema siguiente del WIL puede observarse como fue cayendo la participación del 1\% mas rico de la población en el total de la riqueza en tres de los países con los mayores PBI del planeta y como recuperan una curva ascendente a partir de 1990 (año de la caída de la URSS) acompañados desde entonces también por Rusia y China.

Gráfico 1. Participación del 1\% de mayor riqueza alredor del mundo, 2013-2015: la caída y el incremento de la desigualdad de riqueza personal

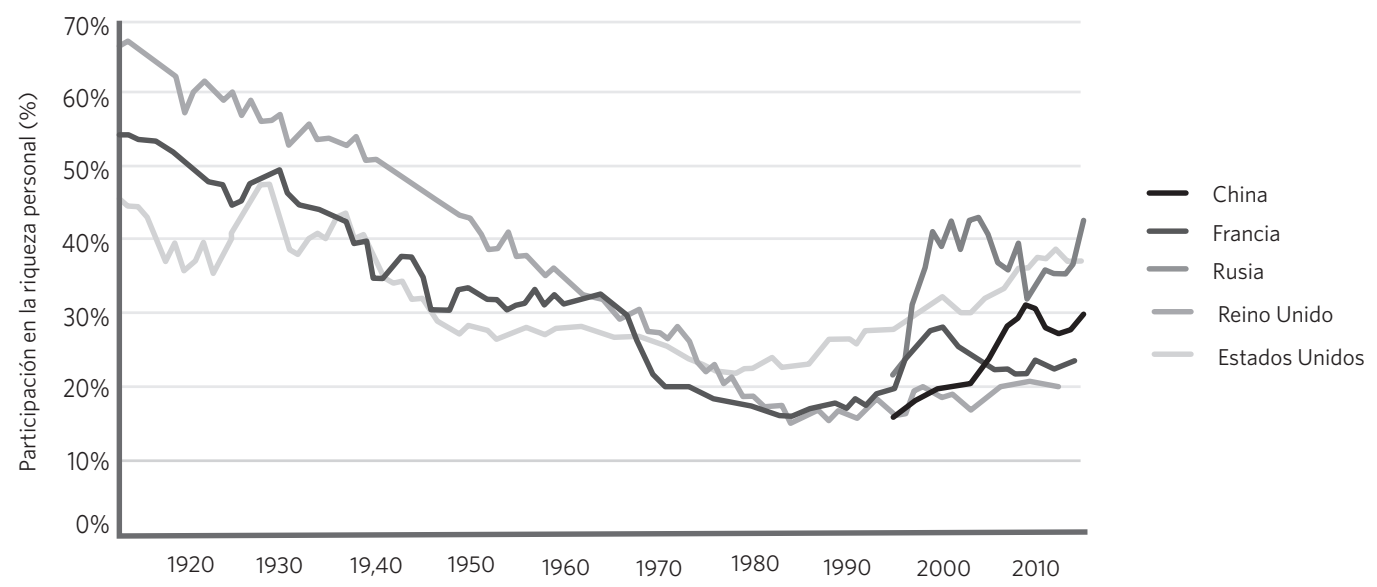

Fuente: World Inequality Report²

Nota: En 2015, la participación del 1\% de mayor riqueza era de 43\% em Rusia, comparado com 22\% em 1995.

Por otro lado, al medir la participación del sector público en el total de la economía en seis países centrales desde 1970 en adelante, el Laboratorio Mundial de la Equidad muestra que esta también fue récord en esa década, pero que no ha dejado de retroceder sistemáticamente en favor del sector privado desde entonces. 
Gráfico 2. El incremento de la riqueza privada y el descenso de la riqueza pública en países ricos, 1970-2016

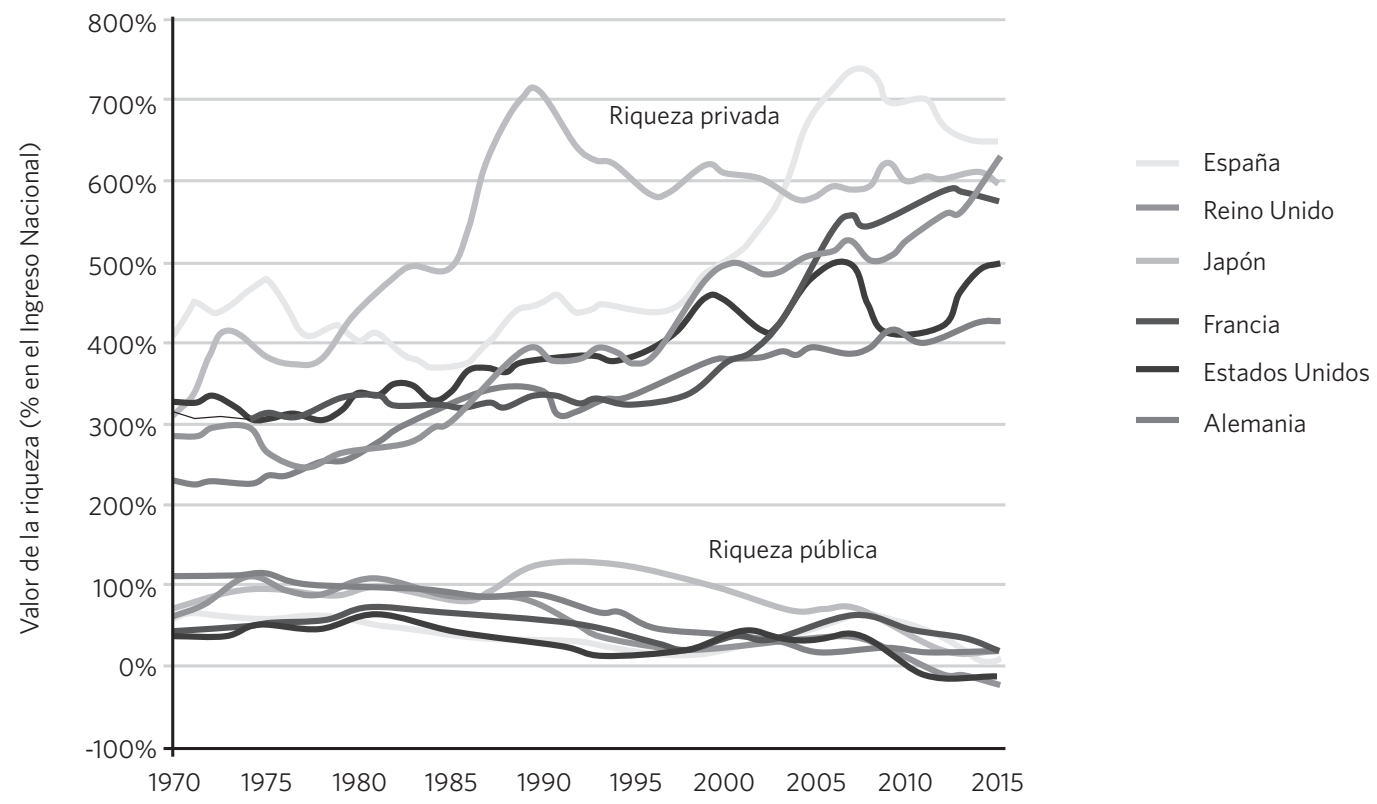

Mientras tanto la desigualdad era y continúa siendo el rasgo distintivo de América Latina.

La declaración de Alma Ata sorprende a la Región en medio de una 'epidemia' de dictaduras con políticas económicas que generan un brutal incremento de la pobreza que llega al final de la década de los 80 a un escalofriante $48,2 \%$ de la población, una situación que comienza a revertirse a comienzos del milenio alcanzando, luego del auge de gobiernos desalineados del Consenso de
Washington, el punto más favorable en el 2014 con un valor que lo reduce al $28,0 \%$ para toda la región. La curva descendente de más de una década se vuelve a revertir en los años más recientes con los dramáticos cambios políticos que han vuelto a promover la concentración del ingreso que se posiciona ahora (2016) en un ascendente valor de $30,7 \%$, lo que puede verse expresado en el siguiente gráfico construido sobre bases estadísticas de la Comisión Económica para América Latina y el Caribe (Cepal) 2017³. 
Gráfico 3. Proporción de la población de América Latina en indigencia y en pobreza

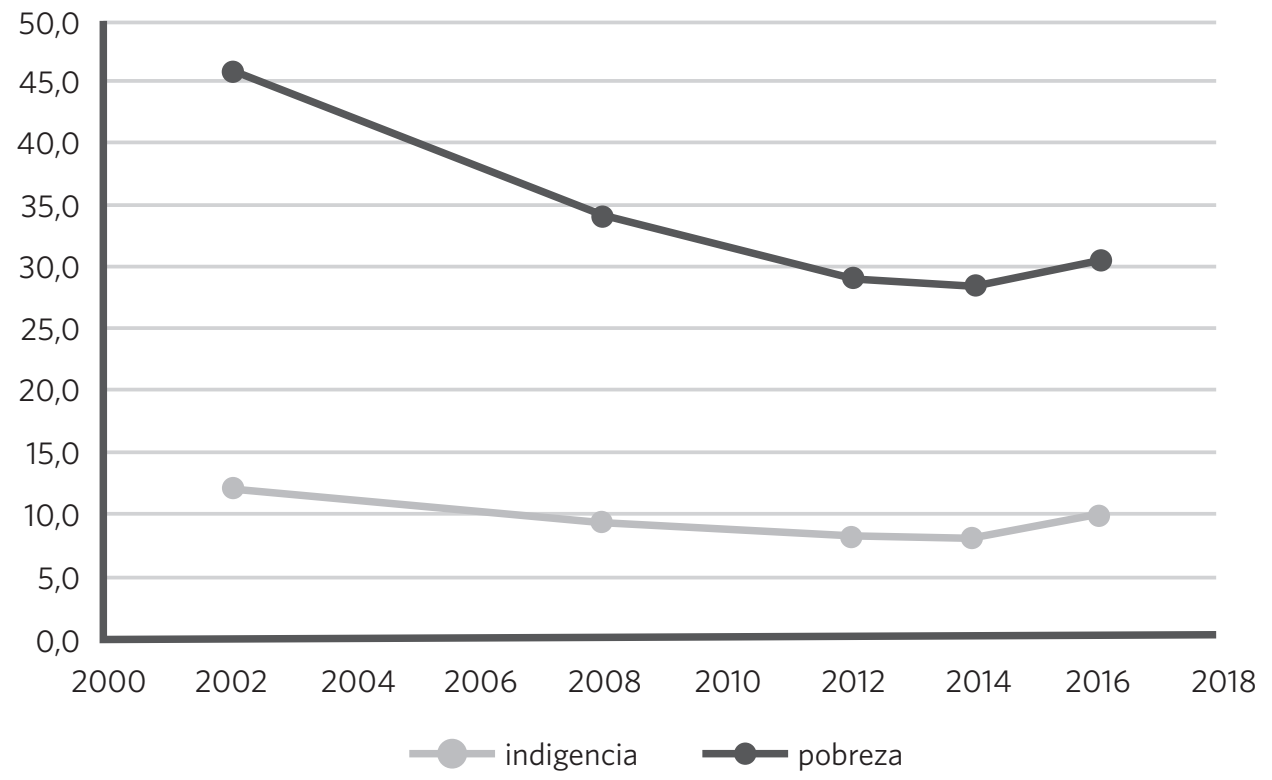

Pero el análisis de la realidad no será lo que amedrente el idealismo estructural de los Organismos Internacionales que se aprestan a aprobar 40 años después, en condiciones por demás desfavorables, un nuevo documento bajo la forma de un borrador de trabajo colgado en el portal de la OMS1(1-2), reafirmando una expectativa de Salud para Todos; y en donde no resulta sencillo sostener lo que en sus propias palabras se afirma: "expresamos nuestra intención de ser la generación que logre la salud para todos [...]" mientras se acelera el camino a la concentración de los ingresos entre y dentro de nuestros países. Tal vez previendo esa objeción el documento define que "los países que implementan exitosamente una APS de calidad obtienen beneficios de salud superiores a lo esperable para su nivel de desarrollo". ¿'Será así?

\section{La APS antes de la APS}

En otros trabajos ${ }^{4}$ hemos explorado cómo las prácticas y la mayoría de los dispositivos de la APS ya estaban disponibles y presentes antes de 1978 y como en buena medida el constructo APS incentivó, amplió, articuló esas experiencias generando un concepto polisémico que logró entramar un conjunto disperso de dispositivos, de escenarios, de modelos de atención e incluso de estrategias populares de supervivencia.

La APS va a reabsorber diversas experiencias, entre otras las de: i. agentes comunitarios de salud, algunos tributarios de las viejas luchas contra los vectores como el caso de la Medicina simplificada de Venezuela, de ii. los centros materno infantiles desplegados en las zonas desfavorecidas de las grandes ciudades, de iii. la fuerte tradición de Gran Bretaña y los países del Commonwealth de los General Practitioners que podríamos denominar la atención 'médica' primaria y de iv. las experiencias mas autogestionarias de poblaciones organizadas dando respuesta a sus problemas más básicos de salud.

Poniendo el centro de gravedad en las formas históricas; el capitalismo, el imperialismo, el neocolonialismo y, más recientemente, el capitalismo globalizado todas a su manera producen muertes y enfermedades 
evitables (aunque también hayan invertido e inviertan ingentes recursos en descubrir tecnologías para remediarlo o cronificarlo claro, cobrando por ello).

Por su parte la medicina social trató con cierto desdén esta estrategia a pesar que, por su ubicación geográfica y geopolítica la reunión de Alma Ata constituyó todo un desafío en esa peculiar etapa de la guerra fría (la derecha la atacó por ser de izquierda pero la izquierda no se la apropió por ser socialdemócrata y/o tercermundista).

\section{La ambivalencia de un constructo que al mismo tiempo es su fortaleza}

Múltiples esfuerzos se han realizado para atrapar el concepto de APS en dimensiones muy disímiles entre sí. ¿̇e trata de un paquete de prestaciones de alta relación costo-efectividad que puede mejorar los indicadores de salud aún en ausencia de mejoras sustanciales en las condiciones de vida?, ¿̇se trata de una puerta de entrada y/o un nivel de atención desconcentrado con cierta capacidad resolutiva que le simplifica el acceso a las personas a los servicios de salud?, ¿̇se trata de una estrategia o aun de una agenda de reforma de los sistemas de salud?, ¿̇se trata de un objeto unificador de la agenda de cooperación técnico-financiera de los organismos bi y multilaterales para lograr el desarrollo sanitario de los países más desfavorecidos?. Y quizás la respuesta más sorprendente sea 'todas las anteriores son correctas' lo que nos impulsa a una pregunta que queda sin respuesta y que constituye un rompecabezas para quienes están pensando una segunda declaración de APS 40 años después: ¿Cómo se las arregló un concepto tan polisémico, para expresar tantas cosas al mismo tiempo y lograr que tantas personas la defiendan y la transformen en su propia causa, en su propia bandera?

\section{Alma Ata Un elefante en el bazar de la asistencia técnica tradicional}

La sola exigencia de dar cumplimiento al mandato de concretar el derecho a la salud a amplias capas de la población mundial hasta entonces sin acceso ni cobertura de servicios bajo una perspectiva de derechos, implícito en la meta lanzada un año antes (1977) de 'Salud para Todos en el año 2000' resulta un constructo de una transversalidad y de una amplitud pocas veces vista ya que claramente no resulta ni resultaba sencillo establecer simples ecuaciones de intervención costo-beneficio que dominaban entonces y dominan hoy aún más la escena internacional.

Una serie de rasgos y elementos constitutivos de la salud internacional contribuyen a caracterizar la declaración de Alma Ata y a la misma APS como un dispositivo contracultural en este selecto mundo diplomático.

La Salud Internacional fue matrizada por más de un siglo a partir de la medicina y de la higiene colonial, perfeccionada por los dispositivos neocoloniales de la Fundación Rockefeller que ejerció y aun ejerce enorme influencia en los Organismos Internacionales.

Según Birn 5 los rasgos básicos de la construcción de un tema de agenda de la asistencia técnica en el campo de la 'Ayuda Internacional' para la Fundación Rockefeller eran:

1. Agenda fijada desde el exterior. Los intereses del prestador y no las necesidades locales determinan la cooperación.

2. Incentivos en presupuesto externo aunque la cooperación requiera un financiamiento sustancial de parte de los propios países receptores.

3. Paradigma tecno-biológico. Elección de enfermedades de alta visibilidad y uso de herramientas técnicas estandarizadas, sin importar la locación. 
4. Parámetros apriorísticos que garantizan el éxito de la cooperación: objetivos delimitados en el tiempo y en el espacio (definidos por logros positivos más que por necesidad). Campañas de mediana y gran escala.

5. Profesionales transnacionales. instrumentales y mediadores para construir consenso sobre las agendas y traducir los intereses internacionales a escenarios locales.

La APS no cumplió casi ninguna de estas premisas invitó a definir las agendas en los espacios nacionales y aun en los subnacionales y locales, no se sustentó en créditos ni requirió costosas y condicionantes cooperaciones, no se concentró en daños o enfermedades sino en poblaciones en riesgo social, (hoy re-nominadas como 'ubatendidas'), no definió plazos ni fechas límites, excepto la meta marco de Salud para Todos en el año 2000, ni requirió importante presencia de expertos internacionales que por cierto no abundan cuando se trata de aportar a una agenda transversal.

\section{0 años en los que pasó de todo}

Es imposible colocar en pocas palabras los dramáticos cambios que fueron modificando la percepción y las condiciones materiales para esa indirecta advocación al derecho a la salud que fue Salud para Todos, pero algunos datos permiten estructurar una cierta periodización.

Entre 1978 y 1988 podemos hablar de una 'década de expansión' -aunque ya en 1981 se verifica el comienzo de la revolución conservadora de la dupla Reagan Thatcher instalando dramáticos retrocesos que lograrán que se califique por primera vez a una década como una 'década perdida'. Entre 1989 y el 2000 podemos identificar un giro hacia la descentralización (District health o Sistemas Locales de Salud -Silos) que constituyó toda una agenda de resistencia y conservación de logros frente a la segunda 'década perdida'. Es que con la caída del muro de Berlín y el fin de la bipolaridad se asiste a un período de intensa internacionalización del capitalismo con ataques selectivos sobre los Estado Nación (visualizados como potenciales enemigos del libre comercio internacional); Estados nacionales que en muchos países constituían el sujeto principal, no solo de las políticas públicas, sino de la propia gestión de los sistemas nacionales de salud.

La fuerte irrupción del Banco Mundial al campo de salud ya no solo como financiador, sino como agente promotor de 'reformas' con su influyente documento 'invertir en Salud' Washington 1993 intentó borrar todo rastro de la declaración de Alma Ata instalando por primera vez lo que será desde entonces su eje conceptual: el 'subsidio a la demanda' que ha destruido y continúa destruyendo los sistemas de salud de numerosos países del mundo, teniendo en nuestra región a Colombia y Chile como sus principales laboratorios de aplicación.

Desde comienzos del siglo XXI, de la mano de estrepitosos fracasos de las políticas neoliberales impulsadas por el consenso de Washington, un grupo de países de América Latina: Venezuela, Brasil, Uruguay, Argentina, Bolivia, Ecuador, Paraguay, El Salvador, Nicaragua deciden desalinearse de ese Consenso e instalar políticas económicas y sociales orientadas hacia una fuerte redistribución del ingreso, lo que en el orden mediático de los países centrales se descalifica con el término 'países con gobiernos populistas'.

Consistente con una lógica de determinación social se produce un descenso para la región del $48 \%$ al $28 \%$ en la proporción de pobres que necesariamente tenía que tener un impacto en la salud de la población propuesta.

En una publicación de Fondo de las Naciones Unidas para la Infancia (Unicef) ${ }^{6}$ pueden verse las modificaciones en la mortalidad de menores de 5 años en los países que desafíaron el modelo del Consenso de Washington y el impacto de las políticas económicas y sociales redistributivas. 
Gráfico 4. Reducción de Mortalidad de Menores de 5 años en países seleccionados

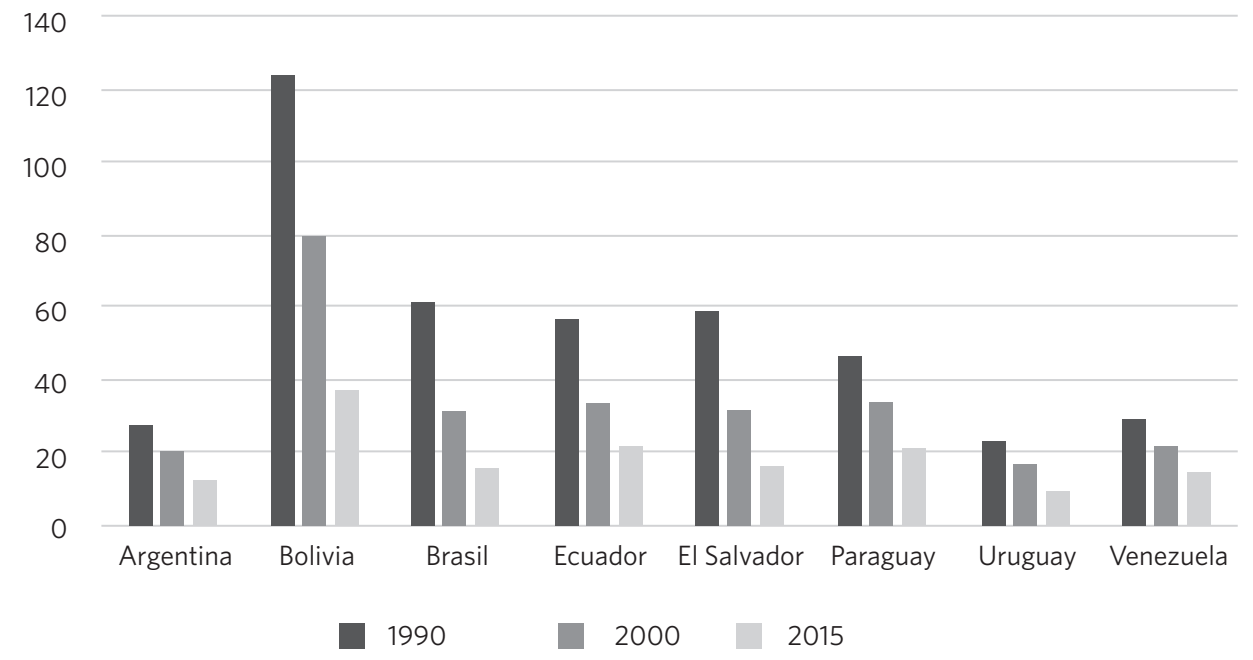

\section{8 frente al 2018: un puñado de similitudes, un océano de diferencias}

Por lo que hemos descripto sumariamente, las condiciones para una declaración no podrían ser más diferentes. El mundo de 1978 se caracterizaba por una marcada bipolaridad que generaba un espacio tanto para la social democracia europea como para un tercer mundo con un fuerte bloque de no alineados, fortalecido por un cierto éxito de la constitución de clubes de exportadores de materias primas que, imitando a la Opep (Organización de Países Exportadores de Petróleo), abría la expectativa de un Nuevo Orden Internacional, una formulación expresamente mencionada en la Declaración de Alma Ata.

Del mismo modo, las conversaciones del denominado Salt II generaban la posibilidad de interrumpir la Carrera armamentista y derivar una parte de esos recursos económicos liberados al desarrollo social y a la salud.

Estados Unidos durante el gobierno de Jimmy Carter había abrazado la causa internacional de los derechos humanos, importante cambio que desubicó a las dictaduras militares del Cono Sur y el representante del Gobierno de ese país, el demócrata Edward Kennedy, contribuyó con su presencia a la visibilidad de la reunión de Alma Ata.

40 años después, el mundo no podría ser más diferente. Un mundo unipolar con un Estados Unidos impredecible y una Europa que se encuentra en pleno desmantelamiento de su Estado de Bienestar y de una privatización progresiva de sus sistemas nacionales de salud, aunque con incipientes reacciones contra la Unión Europea - el principal promotor de las privatizaciones- de parte de los gobiernos de Gran Bretaña (BREXIT), de Italia y de buena parte de la opinión pública de Grecia, España, Bélgica o Portugal.

Por su parte, los Organismos Internacionales de Crédito(WB IMF) y las ONG'sy Fundaciones filantrópicas tienen hoy mayor influencia en salud que los mismos organismos multilaterales especializados del sistema de Naciones Unidas. Según sus propios datos para el 2015 apenas un cuarto de los recursos financieros de la OMS provenían de aportes gubernamentales, mientras un $75 \%$ provenían de laboratorios farmacéuticos, otras empresas trasnacionales y la filantropía internacional. 


\section{La memoria no es tan usual en los Organismos Internacionales}

A pesar de parecer que nos encontramos frente al simple recuerdo o a un homenaje a una reunión más, vale la pena precisar que no es habitual que los organismos internacionales recuerden aniversarios. Son infinidad las reuniones y las declaraciones que se generan y que nunca son rememoradas como para preguntarse por qué Alma Ata ha adquirido tal notoriedad(que hace que pueda generar 'remakes' a 25 años (la Organización Panamericana de la Salud - OPS), a 30 años (la OMS) y ahora a 40 años (OMS-Unicef).

Quizás porque fue parte de un momento histórico único e irrepetible, Quizás porque la producción de la reunión de Alma Ata fue resistida y atacada desde un primer momento, quizás porque interpela a cientos de miles de trabajadores de salud en todo el mundo, quizás porque pocas veces una iniciativa de la OMS y el Unicef adquirió tanto de esa elusiva materia prima que llaman 'consenso'. En todo caso, no cabe dudas que no se trata de un homenaje sino de una instrumentación de este cúmulo que hoy se reúne bajo el nombre APS para su aprovechamiento en iniciativas futuras.

\section{Actoría, o cuando los actores reescriben el guion}

Durante el siglo XV en(el) que la labor y el prestigio de los autores teatrales y literatos como William Shakespeare era(n) reducido(s) se tenía en cambio en gran estima a las Compañías Teatrales, las cuales interpretaban libremente guiones muy generales haciendo que la 'actoría' fuera mas importante que la autoría. Del mismo modo, parece que la vivencia de cientos de miles de trabajadores en el mundo ha reescrito y continúa reescribiendo los guiones de la atención primaria, en cada momento, en cada contexto, en cada situación concreta. Algunos ejemplos, como la abogacía por el derecho a la Salud desarrollada con sólidas raíces institucionales en El Salvador o en la India, los dispositivos de participación social en la toma de decisiones de la política pública, como en Brasil o en Bolivia, la medicalización de la APS que siguiendo la tradición inglesa de los General Practitioners en el Reino Unido se extiende a países tan diversos como Australia, España o Canadá. El sostenimiento de la Enfermería Comunitaria, la experiencia de trabajadores comunitarios de salud reactualizando las históricas experiencias de los feldsher rusos, las experiencias argelinas o la medicina simplificada venezolana.

El amplio espacio que va de la atención hospitalaria hasta lo que hemos denominado el nivel 0 , traducido como el lugar en donde la gente vive estudia trabaja se recrea, se ha poblado de médicos especialistas o generalistas, de enfermeras, de psicólogos, de trabajadores sociales, de odontólogos, de trabajadores comunitarios que aunque en diferentes proporciones y con diferente peso en cada país o región concreta promueven el derecho a la salud junto a las comunidades a las que sirven.

\section{Lo general, lo particular y lo singular}

Citando la desafiante frase de Boaventura Santos "Tenemos el derecho a ser iguales cuando la diferencia nos inferioriza, tenemos el derecho a ser diferentes, cuando la igualdad nos descaracteriza [o desnaturaliza]"7(1) resulta revelador verificar hasta qué punto la APS, desde sus inicios, pero muy especialmente luego de 1986 en que se repliega hacia formas más descentralizadas (district health o sistemas locales de salud), logra instalar una amplia diversidad de experiencias que resisten las taxonomías o las catalogaciones simplistas. En una época de 
crisis de los grandes relatos, intencionalmente o no, al apelar fuertemente a lo local desde fines de los 80 s se rescató la singularidad que hizo brotar el derecho a ser diferente por la constelación de rasgos de culturas, de historias, de habitats de los diferentes conjuntos humanos hasta exhibir una variabilidad casi infinita.

En otras palabras, la pretensión de universalidad de la medicina y de la salud pública occidentales chocó con la realidad de múltiples singularidades, no reducibles a paquetes ni a respuestas protocolizadas o estandarizadas, lo que complejizó fuertemente las aspiraciones de una cooperación técnicofinanciera internacional en salud.

Por eso, dimensiones como lo intercultural o la significación de lo ambiental, aparecen muy negados en el contexto de esa medicina organicista, dimensiones que reemergen con fuerza en la APS haciendo de cada práctica local una experiencia única.

\section{Lo social como lo que explica, pero además como lo debe ser explicado}

La interfase salud sociedad no es una relación subordinada en la que una aparece como la variable dependiente (Salud) y la otra independiente (Sociedad). Lo social está hecho del mundo, de la constelación, de las relaciones interpersonales y los vínculos de atención y de cuidado que están en el centro de las relaciones interpersonales e intergeneracionales y contribuyen centralmente a constituir esa elusiva materia prima que llamamos 'lo social'.

Cuando miembros de una comunidad se capacitan en atención y cuidado de la salud adquieren una cierta autoridad o influencia sobre sus pares que se pone de manifiesto en situaciones de necesidad y constituyen lo que Foucault denominó el 'poder pastoral'. De esta manera la dinámica socio-sanitaria constituye una interacción de doble vía que permite comprender mejor el marco de la determinación del proceso salud-enfermedad-atención-cuidado históricamente construido en cada contexto o realidad concreta.

\section{Finalmente aparecieron los cocodrillos}

A pesar de la convicción sobre la necesidad de comprender los fenómenos complejos por fuera de las historias personales resulta imposible eludir la mención de la figura de Halfdan Mahler a quien se atribuye el ser el salubrista más influyente del siglo XX. Este médico danés con una larga trayectoria en cooperación internacional fue el tercer Director de la OMS por 15 años en el extenso período que va de 1973 a 1988 y fue la figura visible de la adopción de la meta Salud para Todos en el año 2000 de la convocatoria y presidencia de la Conferencia de Alma Ata y de la Carta de Ottawa.

A partir del año 2000, convencido que la APS había fracasado en lograr la meta Salud para Todos y dela necesidad de involucrar progresivamente a las poblaciones en el logro del derecho a la salud, forma parte del núcleo fundador del Movimiento por la Salud de los Pueblos que constituye su primera Asamblea en Bangladesh.

Los principios que rigen el movimiento resultan por demás elocuentes:

- El logro del más alto nivel de salud y bienestar es un derecho humano fundamental, sin importar el color de la persona, su etnia, religión, género, edad, habilidades, orientación sexual o clase.

- Los principios de una APS universal e incluyente - concebidos en la Declaración de Alma Ata de 1978 - deben ser la base para formular las políticas relacionadas con la salud. Más que nunca se requiere de un enfoque equitativo, participativo e intersectorial. 
- Los gobiernos tienen la responsabilidad fundamental de garantizar el acceso universal a una atención en salud de buena calidad, a educación y otros servicios sociales de acuerdo a las necesidades de la población, no a su capacidad de pago.

- La participación de la población y de las organizaciones populares es esencial para la formulación, implementación y evaluación de todas las políticas y programas sociales y de salud.

- La salud es determinada primordialmente por el ambiente político, económico, social y físico y debe ser de alta prioridad al elaborarse las políticas locales, nacionales e internacionales, junto con los temas de equidad y desarrollo sostenible.

El intento de absorber lo que hay construido y se ha acumulado tras el concepto de APS por parte de los privatizadores abiertos o encubiertos de la salud quedó metafóricamente expuesto por Halfdan Mahler8(1) que como presidente honorario de la conferencia de Buenos Aires, (2007) en su discurso inaugural afirmó que: "estamos hasta el cuello en el fango verbal contra los cocodrilos conceptuales, que nos quieren comer de un solo bocado". Y señaló:

Mi profunda esperanza es que esta reunión técnica y política nos proporcione municiones para eliminar los cocodrilos conceptuales que rondan el entorno sanitario.

¿Quienes son o eran para Mahler estos simpáticos, aunque algo agresivos reptiles? Parece que se refiere a:

- El Banco Mundial y el Fondo Monetario Internacional promotores de la privatización creciente de los sistemas públicos de salud a través de la separación de los roles prestador y financiador del Estado y la Seguridad Social.
- La Fundación Rockfeller y la propia Unicef desde la reunión de Bellagio, fuertes propiciadores de una APS selectiva.

- Las Fundaciones filantrópicas y las empresas operando por dentro de la misma OMS torciendo la voluntad de los propios países miembros.

- El Complejo Médico Industrial trasnacionalizado que 'ha descubierto' la APS como una nueva y promisoria fuente de inversiones rentables a partir del incremento de las enfermedades crónicas.

\section{A modo de conclusión}

La APS constituye uno de los más notables giros en la cooperación técnica internacional generadora de un amplio espacio de políticas públicas y una amplia gama de experiencias autogestionarias. En todos los casos se trata de una enorme extensión de cobertura por mecanismos más ortodoxos o heterodoxos de concretar esa cobertura y generador de múltiples diversidades e identidades que emergen a cada paso que la APS logra avanzar en el contacto con poblaciones sub-atendidas.

Como todo proceso de gran escala se presta a infinidad de lecturas e interpretaciones que van desde las mas benignas adjudicándole resultados palmariamente visibles en reducción de mortalidad y cobertura de enfermedades inmuno prevenibles, muestra resultados mucho menos espectaculares en transformaciones ambientales y sociales y definitivamente ha generado mecanismos de sobre-medicalización y disrupción de las estructuras tradicionales, en algunos casos avasallando culturas y arrinconando y agrediendo a las medicinas tradicionales que desde todo punto de vista constituyen al igual que muchas lenguas y culturas en riesgo de extinción un patrimonio de la humanidad. 
Así la APS requiere explicitar adecuadamente las opciones disponibles y las ambigüedades que requieren ser despejadas para reemerger con sus mejores rasgos: comprensiva, participativa, culturalmente respetuosa, intersectorial, y flexible a necesidades cambiantes y abierta a desarrollos tecnológicos pertinentes y socialmente aceptados.

\section{En la encrucijada, se puede elegir el camino de la equidad}

La APS del siglo XXI puede y debe reasumir su rol histórico de paciente y firme constructora de las condiciones para el efectivo ejercicio del derecho a la salud basado en sistemas nacionales de salud de acceso, cobertura y capacidad resolutiva universales, basado en el financiamiento de rentas generales con una fina sintonía con las diversas necesidades, culturas y experiencias locales y una amplia participación social en la planificación y en la evaluación de los servicios para prevenir los clásicos fenómenos de burocratización

Resulta imprescindible neutralizar a quienes quieren subsumirla en las operaciones del Complejo Médico Industrial que con la complicidad de los organismos internacionales de crédito operan escudados en la engañosa formulación de Cobertura Universal de Salud (CUS) que pretende reducir el rol del Estado a un mero 'recaudador y distribuidor de fondos' en última instancia para la expansión de la frontera de financiamiento de un subsector privado concentrado y transnacionalizado, socio estratégico a nivel nacional de ese movimiento.

La constitución de una creciente participación ampliamente informada de la población y de los trabajadores de salud en una alianza sinérgica por el derecho a la salud puede compensar la avanzada de 'los cocodrilos conceptuales' y algunos no tan conceptuales, que pretenden canibalizarla. 


\section{Referencias}

1. World Health Organization. Borrador de Declaración Segunda Conferencia Internacional sobre Atención Primaria de la Salud: Hacia la Cobertura Universal de Salud y el Desarrollo Sostenible Astaná, Kazajstán 25-26 de octubre de 2018. [acceso en 2018 mayo 20]. Disponible en: http://www. who.int/primary-health/conference-phc/DRAFT-Declaration-9-April-2018.pdf.

2. World Inequality Lab: Informe sobre la desigualdad global 2018. [acceso en 2018 mayo 20]. Disponible en: http://wir2018.wid.world/files/download/ wir2018-summary-spanish.pdf.

3. Comisíon Económica para América Latina y el Caribe. Anuario Estadístico de América Latina y El Caribe, 2017 [internet]. [acceso en 2018 ago 29]. Disponible en: http://interwp.cepal. org/anuario_estadistico/Anuario_2017/index. asp?anuario $=2017$ \&idioma $=$ sp.

4. Rovere M. Atención Primaria de la Salud en Debate Revista Saúde debate. 2012 jul-set.; 36(94):327-342.

5. Birn A. Marriage of Convenience. Nova Iorque: University of Rochester Press; 2006.
6. United Nation Children's Fund. Levels \& Trends in Child Mortality report [internet]. 2015. [acceso en 2018 agosto 30]. Disponible en: https://www.unicef. org/spanish/childsurvival/files/IGME_Report_Final2.pdf.

7. Santos BS. Los Derechos Humanos y el Foro Social Mundial [internet]. Quito: Institute de Drets Human de Catalunya; 2004. [acceso en 2018 agosto 30]. Disponible en: http://aplicaciones2.colombiaaprende.edu.co/red_privada/sites/default/files/ de_los_derechos_humanos_y_foros_social_mundial____soussa_santos.pdf.

8. La Nación. Acusaciones sobre Fracasos en Salud [internet]. 2007. [acceso en 2018 agosto 30]. Disponible en: https://www.lanacion.com.ar/934361-acusaciones-sobre-fracasos-en-salud.

Recibido en 18/06/ 2018

Aprobado en 27/08/2018

Conflicto de intereses: inexistente

Apoyo financiero: no hubo 